\title{
Evaluation of Hindlimb Deformity and Posture in Dogs with Grade 2 Medial Patellar Luxation during Awake Computed Tomography Imaging while Standing
}

\author{
Yuma Tomo ${ }^{1}$ Kazuya Edamura ${ }^{1}$ Atsushi Yamazaki ${ }^{1}$ Koji Tanegashima ${ }^{1}$ Mamiko Seki ${ }^{1}$ \\ Kazushi Asano ${ }^{1}$ Selena Tinga ${ }^{2}$ Kei Hayashi ${ }^{2}$ \\ ${ }^{1}$ Laboratory of Veterinary Surgery, Department of Veterinary \\ Medicine, College of Bioresource and Sciences, Nihon University, \\ Fujisawa, Kanagawa, Japan \\ 2 Department of Clinical Sciences, College of Veterinary Medicine, \\ Cornell University, Ithaca, New York, United States

\begin{abstract}
Address for correspondence Kazuya Edamura, PhD, DVM, Diplomate JCVS, Laboratory of Veterinary Surgery, Department of Veterinary Medicine, College of Bioresource Sciences, Nihon University, 1866 Kameino, Fujisawa, Kanagawa 252-0880, Japan
\end{abstract} \\ (e-mail: edamura.kazuya@nihon-u.ac.jp).
}

Vet Comp Orthop Traumatol 2022;35:143-151.

\begin{abstract}
Keywords

- bone deformities

- computed tomography

- dogs

- patellar luxation

- postural abnormalities

Objective The aim of this study was to determine the degree of bone deformities and hindlimb postural abnormalities in a standing position in awake Toy poodles with and without grade 2 medial patellar luxation (MPL) using high speed 320-row computed tomography (CT).

Methods The limbs with grade 2 MPL (MPL-G2 group) and without any orthopaedic disorders (control group) were imaged in a standing position, without sedation or anaesthesia, using CT. In MPL-G2 group, images were obtained when the patella was luxated (G2-L group) and reduced (non-luxation, G2-NL group). Bone morphologies of the femur and tibia were quantified three-dimensionally. Hindlimb standing posture was evaluated by measuring femoral rotation and abduction angles, tibial rotation angle, metatarsal rotation angle, foot rotation angle, angle between the femoral anatomical axis and the mechanical axis of hindlimb and stifle joint line convergence angle.

Results There were no significant differences in bone morphologic parameters between the MPL-G2 group (5 limbs) and the control group (6 limbs). In the G2-NL group, there were no significant hindlimb postural abnormalities. In contrast, in the G2L group, significant hindlimb postural abnormalities including external rotation of femur, internal rotation of tibia and foot, external rotation of tarsal joint, large stifle joint convergence angle, genu varum and toe-in standing were observed.

Conclusion Dogs with grade 2 MPL have no bone deformities but show abnormal standing posture when the patella is luxated.
\end{abstract}

received

April 28, 2021

accepted after revision

October 18, 2021

published online

December 21, 2021
DOI https://doi.org/

10.1055/s-0041-1740607.

ISSN $0932-0814$. (c) 2021. The Author(s).

This is an open access article published by Thieme under the terms of the Creative Commons Attribution-NonDerivative-NonCommercial-License, permitting copying and reproduction so long as the original work is given appropriate credit. Contents may not be used for commercial purposes, or adapted, remixed, transformed or built upon. (https://creativecommons.org/ licenses/by-nc-nd/4.0/)

Georg Thieme Verlag KG, Rüdigerstraße 14, 70469 Stuttgart, Germany 


\section{Introduction}

Medial patellar luxation (MPL) is one of the most common causes of hindlimb lameness in dogs. ${ }^{1,2}$ It has been reported that MPL can lead to varying degrees of bone deformities of both the femur and tibia, depending on the severity of the MPL. ${ }^{3-5}$ In general, the bone deformities associated with MPL are evaluated with radiographs, but recently three-dimensional computed tomography (3D-CT) has been utilized for more accurate evaluation. ${ }^{5}$ In our previous study using 3D-CT, the dogs with grade 4 MPL had significant femoral varus deformity, medial displacement of the tibial tuberosity, internal torsion of the proximal tibia and hypoplasia of the patella. ${ }^{5}$ In contrast, the dogs with grade 2 MPL showed no significant bone deformities in our previous study using 3D-CT. ${ }^{5}$ Howev$\mathrm{er}$, in the clinical situation, abnormal hindlimb posture including genu varum is often observed in dogs with grade 2 MPL. ${ }^{6}$

In human medicine, the postural abnormalities of the lower limb can be detected with radiographs using full-length standing anteroposterior radiographs from hip to ankle to assess lower limb alignment. ${ }^{7,8}$ In dogs, the ability to make full length radiographs during standing is prohibited by the position of the torso. Thus, it is difficult to evaluate for postural abnormalities of limbs in the standing position with conventional radiography. There has been a report on the evaluation of forelimb alignment using standing radiography in dogs. ${ }^{9}$ However, this report evaluated only elbow and carpal joints, not the entire limb and did not evaluate hindlimb. ${ }^{9}$

Recent technological innovations have greatly reduced the time required for $\mathrm{CT}$ scans, making it possible to perform scans within 3 to 5 seconds in small breed dogs. If CT images could be obtained in awake, standing dogs, without anaesthesia or sedation, the natural standing limb posture could be evaluated in three dimensions. The purpose of this study is to evaluate hindlimb deformity and posture in awake dogs with and without grade 2 MPL while standing naturally using innovative high speed 320-row CT imaging. We hypothesized that the dogs with grade 2 MPL would have significant hindlimb postural abnormalities while standing.

\section{Materials and Methods}

\section{Case Selection}

Toy poodles with a diagnosis of grade 2 MPL (MPL-G2 group) that could stand up with all four limbs on the ground were included. $^{2}$ Dogs with any orthopaedic conditions other than grade 2 MPL were excluded from this study. Toy poodles without any orthopaedic conditions were included as controls (control group). Signalment data including age, gender, body weight, body condition score (BCS, 5-point system) and lameness score (numerical rating score) for all subject dogs were collected from the medical record. ${ }^{10,11}$ This study was approved by the clinical research and trial ethics committee, Animal Medical Center of Nihon University (ANMEC-3-007). In the control group, all owners requested whole body screening for the purpose of a supplement to physical examination using CT without anaesthesia or sedation and consented to the collection of data for this study. In MPL-G2 group, only the caudal half of the body was scanned with CT to avoid excessive radiation exposure.

\section{Awake Computed Tomography Imaging while Standing}

A 320-row area detector CT (Aquilion One, Canon Medical Systems, Co., Otawara, Japan) was used for naturally standing CT imaging. This innovative CT is capable of scanning the entire body at high speed and with low radiation exposure. The subject dog was placed in a clear acrylic case and allowed to stand naturally without any fixation devices (-Fig. 1). Based on a preliminary study, a clear acrylic case with 5 to $10 \mathrm{~cm}$ of space in front, behind, and to each side of the dog was used to allow the dog to stand naturally for awake standing CT imaging. No sedatives or anaesthetics were used for the CT scans.
A

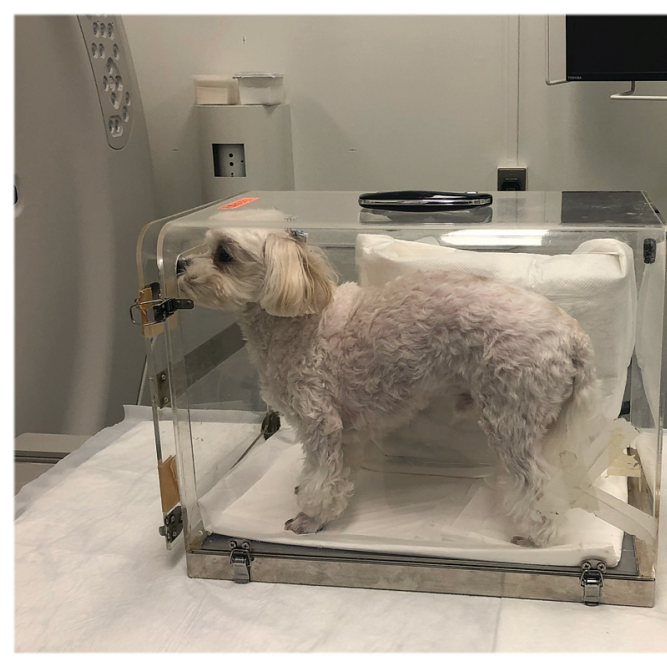

B

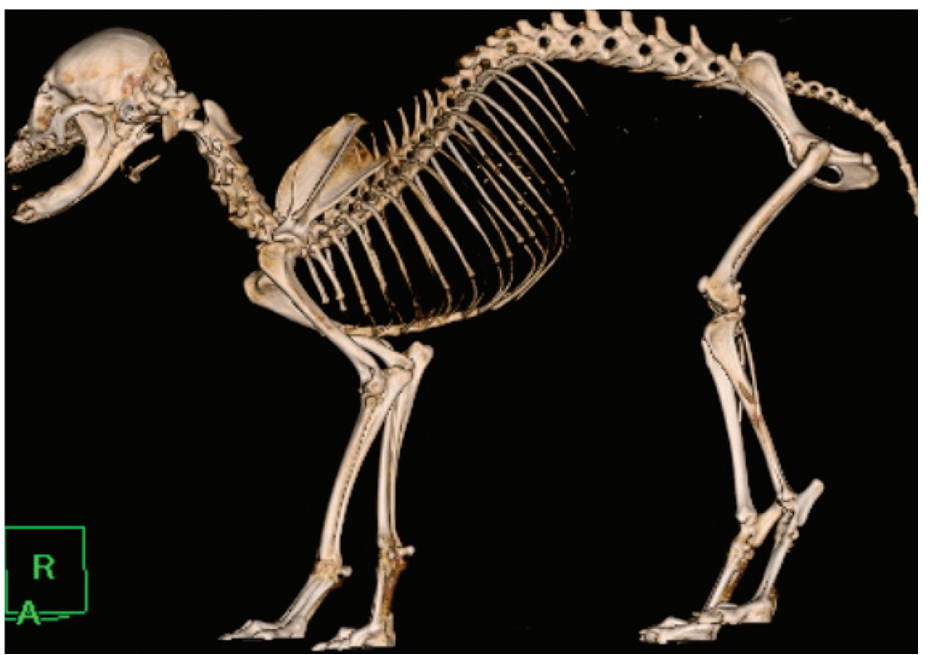

Fig. 1 Method and reconstructed image of awake computed tomography (CT) imaging while standing. (A) The subject dog was placed and stood naturally in a clear acrylic case without any sedation or fixation devices. (B) Three-dimensional reconstructed image of awake CT imaging while standing. 
In the MPL-G2 group, two hindlimb CT scans were performed. On one scan, both patellae were in the reduced position (G2-NL), and on the other scan one patella was in the luxated position (G2-L). The patella was never forced out of or into the trochlear groove; instead, the dogs were asked to sit and stand or move their hindlimb through a natural range of motion until one (left or right, not both) patella was palpated to be in the luxated position. This approach eliminated the need to force the patella into the desired position for imaging. After confirming that the subject dogs were maintaining a standing posture with all four paws on the bottom of the case, CT was taken. All imaging was performed under the following conditions: tube voltage: $120 \mathrm{kV}$; tube current: auto adjustment within the range of 50 to $350 \mathrm{~mA}$; scanning speed: 0.3 to 0.5 seconds; scanning range: $0.5 \mathrm{~mm}$ $\times 80$ row; display field of view: maximum to $500 \times 500 \mathrm{~mm}$; slice thickness: $0.5 \mathrm{~mm}$; reconstruction interval: $0.5 \mathrm{~mm}$. Reconstruction of 3D images and data analysis were performed using image processing software (AZE Virtual Place, AZE Co., Ltd, Tokyo, Japan).

\section{Definition of Each CT View}

Using the 3D models, the frontal view of the femur and the frontal and lateral views of the tibia were generated using the same methods as the previous study. ${ }^{5}$ Three-dimensional images of straight lateral view of the pelvis ( - Fig. 2A) were generated by rotating $3 \mathrm{D}$ reconstruction images, until two reference lines of both left and right sides overlap. The reference line was drawn connecting the following two cranial and caudal landmarks: the tip of the dorsal margin of the ilium (red dot in - Fig. 2A) and the ischial tuberosity (red dot in -Fig. 2A). The dorsal view of the pelvis was then obtained by rotating the lateral view of pelvis 90 degrees to view the dorsal aspect (-Fig. 2B). The caudal view of the

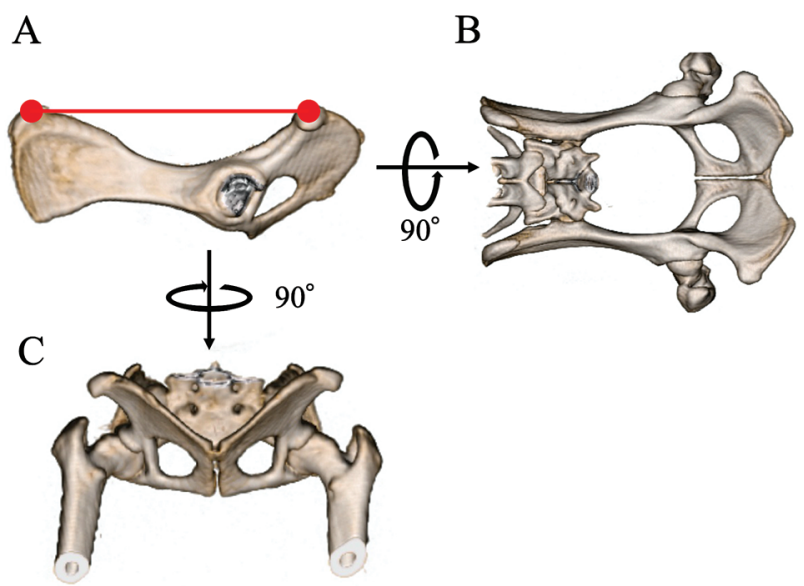

Fig. 2 Lateral, dorsal, and caudal views of the pelvis. (A) Lateral view of the pelvis. The image where the left and right reference lines connecting a tip of a dorsal margin of the ilium (left red dot) and a caudal margin of the ischial tuberosity (right red dot) are overlapping. (B) Dorsal view of the pelvis. The image obtained by rotating 90 degrees from lateral view of the pelvis to view the dorsal side. (C) Caudal view of the pelvis. The view obtained by rotating 90 degrees horizontally from lateral view of the pelvis and viewing from the caudal side. pelvis was obtained by rotating the lateral view 90 degrees to

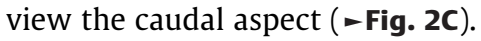

Lateral and dorsal views of the entire articulated hindlimb were also generated to evaluate the posture of the hindlimb. The lateral view was defined as the view where the most cranial margins of ilium overlapped ( - Fig. 3A). The dorsal view was defined by the plane parallel to the surface on which the dogs was standing (-Fig. 3B). In addition, transverse views of the stifle and tarsal joints were defined as the plane parallel to the standing surface in the respective joint region of entire hindlimb image ( - Fig. 3C, D).

Lastly, proximal and distal frontal views of the tibia were obtained to evaluate the alignment of the femur and tibia.

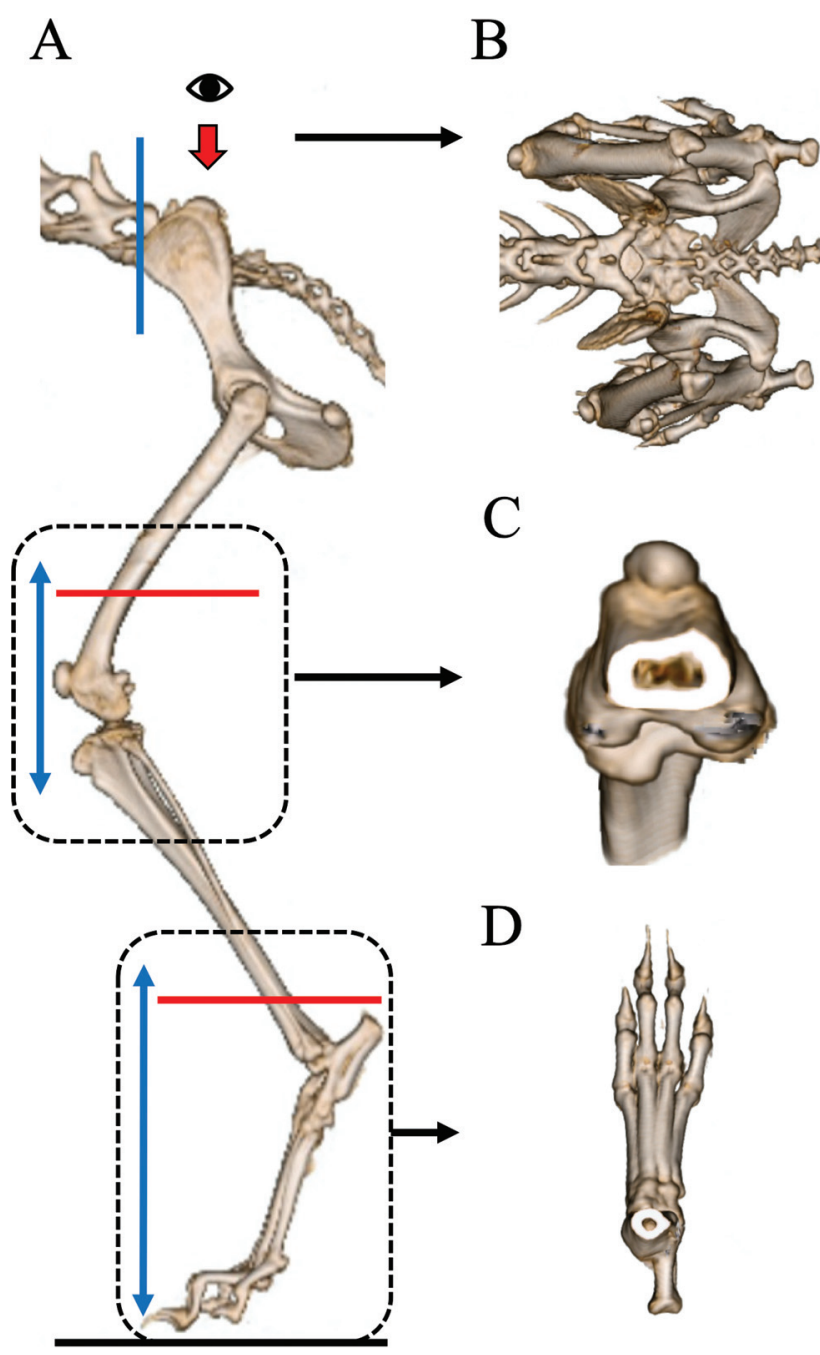

Fig. 3 The lateral and dorsal views of entire hindlimb and transverse views of stifle and tarsal joints. (A) Lateral view of the entire hindlimb. This view was obtained by observing hindlimb from immediately lateral side at the position where the most cranial margins of ilium overlapped (blue line). (B) Dorsal view of the entire hindlimb. The view was obtained by observing from dorsal side in the plane parallel to the surface where dogs standing (eye marking and red arrow in A). (C) Transverse view of the stifle joint. The view parallel to the standing surface (red line) in the stifle joint region (black dotted line and double arrow blue line) of entire hindlimb image. (D) Transverse view of the tarsal joint. The view parallel to the standing surface (red line) in the tarsal joint region (black dotted line and double arrow blue line) of entire hindlimb image. 
A
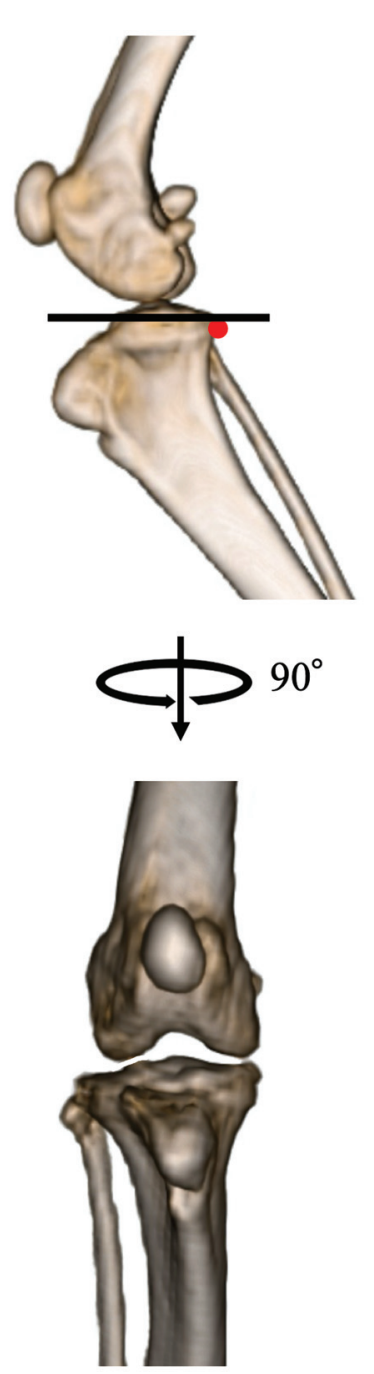

B

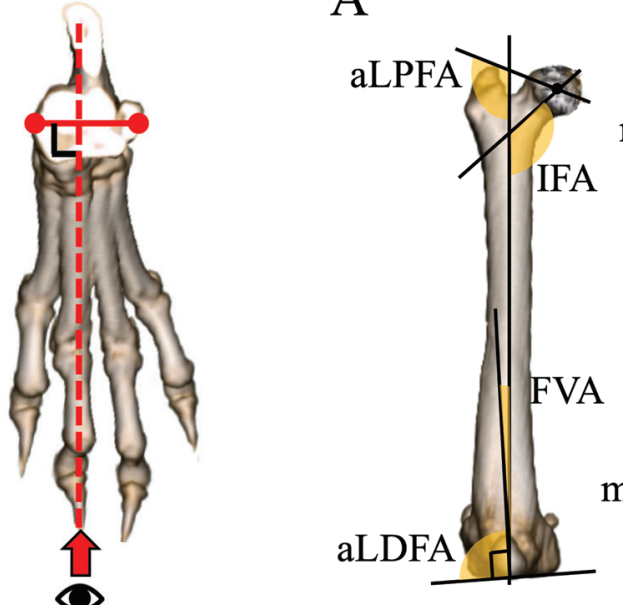

B

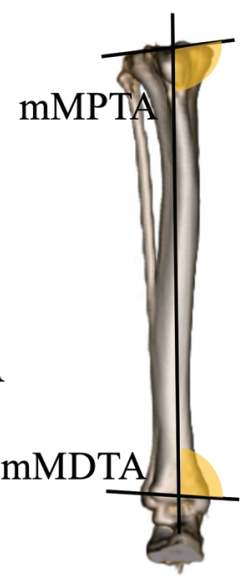

C

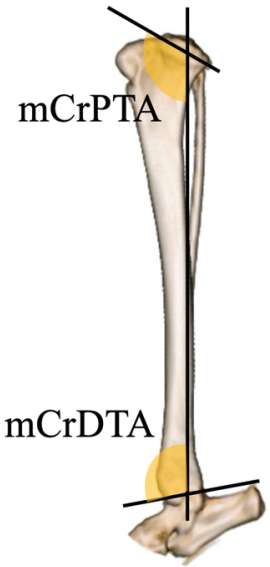

Fig. 5 Measurement values for the bone morphologies of the femur and tibia. (A) Anatomical lateral proximal femoral angle (aLPFA), anatomical lateral distal femoral angle (aLDFA), inclination of the femoral head angle (IFA) and femoral varus angle (FVA). (B) Mechanical medial proximal tibial angle (mMPTA) and mechanical medial distal tibial angle (mMDTA). (C) Mechanical cranial proximal tibial angle (mCrPTA) and mechanical cranial distal tibial angle (mCrDTA).

femoral varus angle were measured on the frontal view of the femur (-Fig. 5A). In addition, anteversion angle (AA) was measured on axial view of the femur. The mechanical medial proximal tibial angle (mMPTA) and the mechanical medial distal tibial angle (MMDTA) were measured on the frontal view of the tibia. The mechanical cranial proximal tibial angle ( $\mathrm{MCrPTA}$ ) and the mechanical cranial distal tibial angle ( $\mathrm{mCrDTA}$ ) were measured on the lateral view of the tibia (-Fig. 5B, C).

\section{Evaluation of Hindlimb Standing Posture}

The flexion/extension angles of the hip, stifle and tarsal joints were measured on the lateral view of the entire hindlimb, according to the method previously reported in dogs (-Fig. 6). ${ }^{13,14}$

The rotation angle and the abduction angle of the femur were measured to evaluate the degree of external rotation and abduction of the hip joint. The femoral rotation angle was measured as the angle formed by the line passing through the spinous process of sacrum and the pubic symphysis (midline of pelvis) and the axis of femoral neck on the dorsal view of the pelvis ( - Fig. 7A). ${ }^{14}$ The femoral abduction angle was measured as the angle formed by the line passing through the center of both femoral heads and the proximal anatomical axis of femur on the caudal view of the pelvis (-Fig. 7B).

The tibial rotation angle and the metatarsal rotation angle were also measured to evaluate the rotational alignment of the stifle and tarsal joints. Tibial rotation angle was measured as the angle formed by the line passing along the caudal edge of medial and lateral femoral condyles and the line passing along the caudal edge of medial and lateral tibial condyles on the transverse view of the stifle joint 


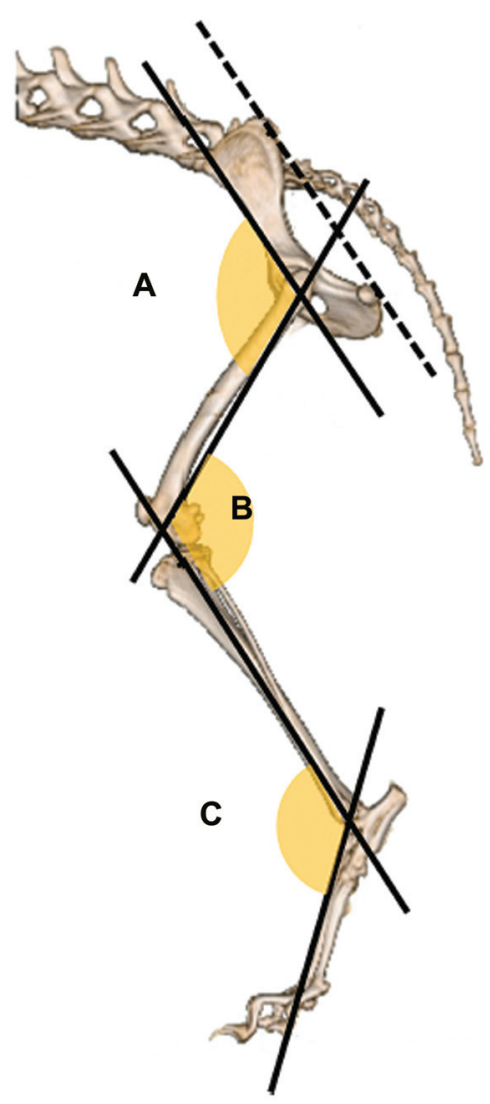

Fig. 6 Flexion/extension angles of the (A) hip, (B) stifle and (C) tarsal joints. An increased value indicates extension.

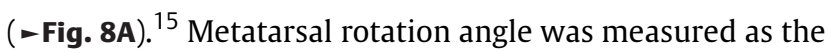
angle formed by the line perpendicular to the line passing through the tips of the medial and lateral malleoli and the line between the third and fourth metatarsal bones (reference line of foot) on the transverse view of the tarsal joint (-Fig. 8B).
A

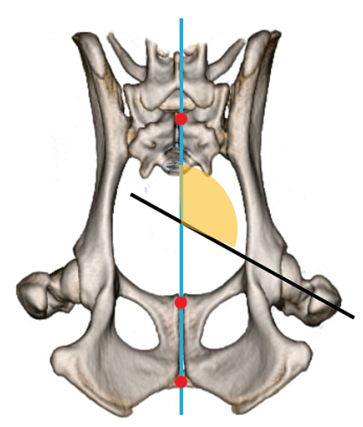

B

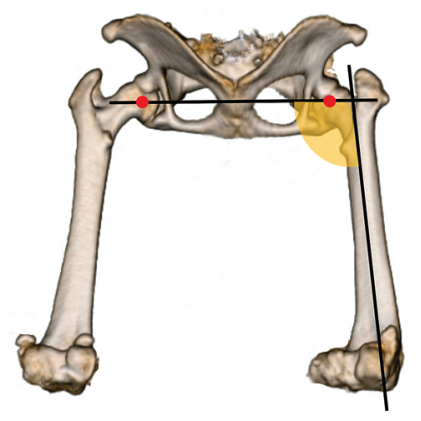

Fig. 7 Femoral rotation and abduction angles. (A) Femoral rotation angle. The angle formed by the line (blue line) passing thorough the spinous process of sacrum (uppermost red dot) and the midline of pelvis (bottom two red dots) and the axis of femoral neck on the dorsal view of the pelvis. An increased value indicates external femoral rotation. (B) Femoral abduction angle. The angle formed by the line passing thorough the center of both femoral head (red dots) and the proximal anatomical axis of femur on the caudal view of the pelvis. An increased value indicates abduction.

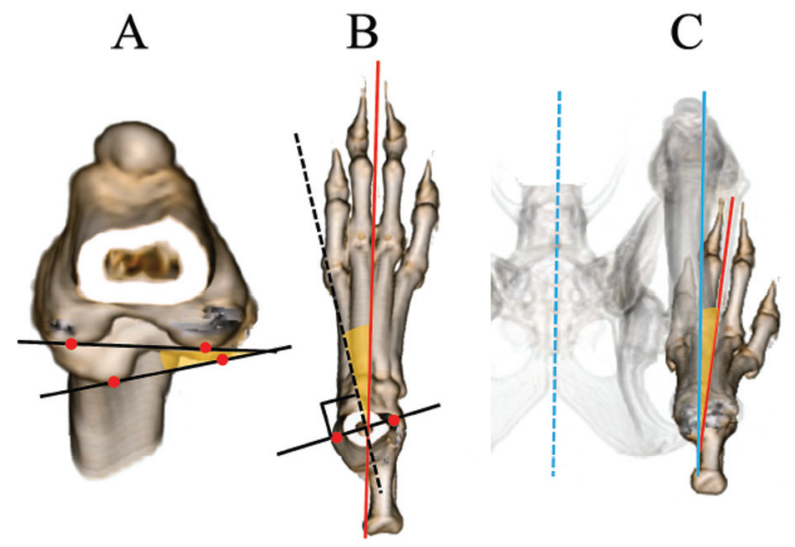

Fig. 8 Tibial, metatarsal and foot rotation angles. (A) Tibial rotation angle. The angle formed by a line passing along the caudal edge of medial and lateral femoral condyles (red dots) and a line passing along the caudal edge of medial and lateral tibial condyles (red dots) on the transverse view of the stifle joint. (B) Metatarsal rotation angle. The angle formed by a line (black dotted line) perpendicular to the line (black line) passing through the tips of the medial and lateral malleoli (red dots) and a line (red line) between the 3rd and 4th metatarsal bones on the transverse view of the tarsal joint. (C) Foot rotation angle. The angle formed by a line (blue line) parallel to the midline of

The foot rotation angle was measured to evaluate the degree of rotation of the entire hindlimb. The foot rotation angle was measured as the angle formed by the line parallel to the midline of pelvis and the reference line of foot on the dorsal view of the entire hindlimb ( $\mathbf{- F i g . ~ 8 C}$ ). Overall hindlimb posture was also assessed as toe-in or toeout posture.

For the purpose of evaluating the degree of genu varum or valgus in the standing position, the angle formed by the
A

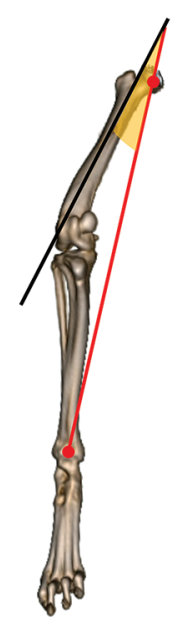

B

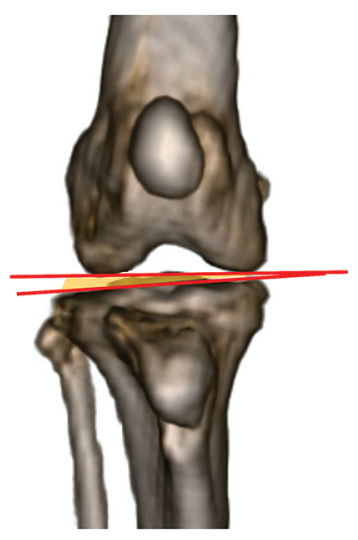

Fig. 9 Angles measured to evaluate genu varum. (A) Angle formed by the proximal anatomical axis of femur and the mechanical axis of hindlimb. The mechanical axis of hindlimb (red line) was defined as the line the line connecting the center of the femoral head (upper red dot) and the midpoint of a line between the medial and lateral malleoli (bottom red dot) on the distal frontal view of the tibia. (B) Stifle joint line convergence angle. The angle formed between distal joint orientation line of the femur and proximal joint orientation line of the tibia on the proximal frontal view of tibia. 
proximal anatomical axis of femur and a line connecting the center of the femoral head and the midpoint of the line connecting the medial and lateral malleoli (mechanical axis of hindlimb) was measured on the distal frontal view of the tibia (-Fig. 9A). ${ }^{16}$ In addition, the stifle joint line convergence angle was the angle formed between distal joint orientation line of the femur and proximal joint orientation line of the tibia on the proximal frontal view of tibia (-Fig. 9B). ${ }^{16,17}$

\section{Statistical Analysis}

The data obtained from the described experiments were indicated as median and range of values. Statistical analyses were performed using data analysis software package (GraphPad Prism version 6.0 for Macintosh, GraphPad Software Inc., San Diego, United States). Mann-Whitney U test was used to compare measurement values between the control group and MPL-G2 group. The data among three groups were tested using Kruskal-Wallis test and Dunn's multiple comparison was used as the post-hoc test. Values of $p<0.05$ indicated a significant difference.

\section{Results}

\section{Patients}

Eleven hindlimbs of 7 Toy poodles were evaluated during this study period. The MPL-G2 group consisted of five hindlimbs in four dogs (Unilateral: $n=3$, bilateral: $n=1$ ). The median age of the dogs was 4.5 years (range from 1 to 8 years) and the median body weight of dogs was $3.0 \mathrm{~kg}$ (range of 2.0 to $5.3 \mathrm{~kg}$ ). The BCS was 4 for one and 3 for the other three. All dogs included in the MPL-G2 group had a lameness score of 1 . The control group consisted of six hindlimbs in three dogs. The median age of the dogs was 11 years (range from 10 to 13 years), and the median body weight of the dogs was $2.3 \mathrm{~kg}$ (range of 2.0 to $2.5 \mathrm{~kg}$ ). The BCS was 3 for one dog and 4 for two dogs. All dogs in the control group had a lameness score of 0 . All dogs included in this study were spayed females.

\section{Bone Morphologies of Femur and Tibia}

There were no significant differences in the bone morphologic parameters of femora between the control and the MPL-G2 groups, including aLPFA, aLDFA, IFA, IVA and AA (-Table 1). Similarly, no significant differences were identified in the bone morphologic parameters of the tibiae between the control and the MPL-G2 groups, including mMPTA, mMDTA, mCrPTA and mCrDTA (-Table 1).

\section{Hindlimb Standing Posture}

There were no significant differences between the control group and the G2-NL group in all measured postural parameters. In contrast, there were significant differences in several postural parameters in the G2-L group compared with not only the control group but also G2-NL group (-Table 2 ).

Flexion/extension angles of the hip, stifle and tarsal joints in the standing position were not significantly different between the three groups. The femoral rotation angle in the G2-L group was significantly higher (external rotation of femur) than that in the other groups. However, there was no significant difference in femoral rotation angle between the control and G2-NL groups. No significant differences were observed in femoral abduction angle among the groups.

The tibial rotation angle in G2-L group was significantly higher (internal rotation of tibia) than that in other groups and the metatarsal rotation angle in G2-L group was significantly higher (external rotation of metatarsal bones) than that in the control group. The foot rotation angle in the G2-L group was significantly lower than that in the control group, and only the G2-L group had negative value, indicating a toein posture (-Fig. 10).

The angle between femoral proximal anatomical axis and the mechanical axis of hindlimb and the stifle joint line convergence angle in the G2-L group were significantly higher than those in other groups, suggesting that the hindlimb in the G2-L group exhibited a significant genu varum posture (-Fig. 10).

Table 1 Measurement values for the bone morphologies of femur and tibia

\begin{tabular}{|l|l|l|l|}
\hline \multirow{5}{*}{ Femur } & & $\begin{array}{l}\text { Control } \\
\text { median (range) }\end{array}$ & $\begin{array}{l}\text { MPL-G2 } \\
\text { median (range) }\end{array}$ \\
\cline { 2 - 4 } & aLPFA & $112.3(110.7-115.3)$ & $114.4(112.4-115.7)$ \\
\cline { 2 - 4 } & aLDFA & $90.3(88.9-91.7)$ & $91.2(90.5-92.8)$ \\
\cline { 2 - 4 } & IFA & $118.9(118.1-120.2)$ & $121.9(118.3-128.2)$ \\
\cline { 2 - 4 } & FVA & $0.7(-1.1-1.7)$ & $1.2(-0.5-2.8)$ \\
\cline { 2 - 4 } & AA & $18.5(16.4-20.0)$ & $17.2(15.8-18.6)$ \\
\hline \multirow{5}{*}{ Tibia } & mMPTA & $94.7(93.9-97.1)$ & $96.3(93.2-100.3)$ \\
\cline { 2 - 4 } & mMDTA & $95.0(92.3-97.7)$ & $93.7(92.9-96.3)$ \\
\cline { 2 - 4 } & mCrPTA & $109.5(108.9-116.8)$ & $112.1(109.5-117.0)$ \\
\cline { 2 - 4 } & mCrDTA & $99.5(94.8-102.1)$ & $101.9(97.9-103.4)$ \\
\hline
\end{tabular}

Abbreviations: AA, anteversion angle; aLDFA, anatomical lateral distal femoral angle; aLPFA, Anatomical lateral proximal femoral angle; FVA, femoral varus angle; IFA, inclination of the femoral head angle; mCrDTA, mechanical cranial distal tibial angle; mCrPTA, Mechanical cranial proximal tibial angle; mMDTA, mechanical medial distal tibial angle; mMPTA, Mechanical medial proximal tibial angle. 
Table 2 Measurement values for standing posture and hindlimb alignment

\begin{tabular}{|l|l|l|l|}
\hline & $\begin{array}{l}\text { Control } \\
\text { median (range) }\end{array}$ & $\begin{array}{l}\text { G2-NL } \\
\text { median (range) }\end{array}$ & $\begin{array}{l}\text { G2-L } \\
\text { median (range) }\end{array}$ \\
\hline Hip joint flexion/extension angle & $105.3(103.2-109.3)$ & $116.4(108.5-116.6)$ & $124.9(97.9-135.0)$ \\
\hline Stifle joint flexion/extension angle & $121.9(93.5-133.7)$ & $123.2(107.9-134.4)$ & $124.8(85.4-128.9)$ \\
\hline Tarsal joint flexion/extension angle & $126.7(110.9-128.7)$ & $132.4(121.3-136.9)$ & $131.2(110.0-139.6)$ \\
\hline Femoral rotation angle & $110.9(94.7-119.1)^{c}$ & $103.1(96.0-119.1)^{c}$ & $123.9(120.3-134.1)^{\mathrm{a}, \mathrm{b}}$ \\
\hline Femoral abduction angle & $99.8(91.3-107.4)$ & $97.4(89.9-97.6)$ & $97.8(95.2-104.6)$ \\
\hline Tibial rotation angle & $5.9(-15.6-10.7)^{\mathrm{c}}$ & $4.8(2.2-11.2)^{\mathrm{c}}$ & $37.5(17.7-44.9)^{\mathrm{a}, \mathrm{b}}$ \\
\hline Metatarsal rotation angle & $1.6(-1.2-5.3)^{\mathrm{c}}$ & $3.0(1.7-10.1)$ & $7.7(4.8-10.5)^{\mathrm{a}}$ \\
\hline Foot rotation angle & $36.1(28.7-69.8)^{\mathrm{c}}$ & $14.4(9.1-35.7)$ & $-0.7(-20.8-8.3)^{\mathrm{a}}$ \\
\hline $\begin{array}{l}\text { Angle of femoral proximal anatomical axis } \\
\text { and mechanical axis of hindlimb }\end{array}$ & $3.0(0.4-5.3)^{\mathrm{c}}$ & $2.5(0.1-5.1)^{\mathrm{c}}$ & $12.6(5.7-18.7)^{\mathrm{a}, \mathrm{b}}$ \\
\hline Stifle joint line convergence angle & $0.7(-2.2-3.5)^{\mathrm{c}}$ & $1.2(0.2-2.3)^{\mathrm{c}}$ & $4.7(3.6-5.0)^{\mathrm{a}, \mathrm{b}}$ \\
\hline
\end{tabular}

Note: Median and range of values in the same row that have superscript lower-case letters are significantly different between MPL grade groups $(p<0.05)$ (a versus control; b versus G2-NL; $c$ versus G2-L).

\section{Discussion}

This study objectively evaluated canine hindlimb morphology and posture three-dimensionally by performing extra high speed, awake standing CT imaging in dogs with and without grade 2 MPL. No bone deformities were observed in dogs with grade 2 MPL compared with the controls, in agreement with the previous study. ${ }^{5}$ In addition, no significant hindlimb postural abnormalities were observed in dogs with grade 2 MPL when the patella was reduced, compared with the control dogs. In contrast, significant hindlimb postural abnormalities including external rotation of femur, internal rotation of tibia and foot, large stifle joint convergence angle, genu varum and toe-in standing were observed in grade 2 MPL dogs when the patella was in the luxated position (-Fig. 10).

In human medicine, standing 2D radiography has been used to evaluate the alignment of the lower limb however, standing CT imaging is preferred for objective evaluation of rotational abnormalities. ${ }^{17,19}$ Quadruped patients have the added challenge of obstruction of the upper limb by the trunk when attempting to use 2D imaging during natural standing. Therefore, we elected to utilize CT scanning, instead of 2D radiography, to report accurate 3D hindlimb postural results during standing. Recently, advances in CT technology have made it possible to obtain images in a short period of time; thus, the opportunities to perform whole body screening by CT without sedatives or anaesthesia have been increased in our teaching hospital. In fact, 320-row area detector CT can scan the whole body of a Toy poodle in approximately 3 to 5 seconds. This allows awake CT imaging while the dog standing without the use of the drugs or fixation devices for immobilization.

It has been reported that severe MPL causes bone deformities as well as abnormal hindlimb posture. ${ }^{2,20}$ Previous CT studies have demonstrated a variety of bone deformities from MPL in both the femur and tibia, and the severity of deformity is related to the severity of the MPL. ${ }^{4,5,21}$ Interestingly, both a recent study and the present study showed that Toy poodles with grade 2 MPL have no significant bone deformities. ${ }^{5}$ However, this present study also demonstrated that dogs with grade 2 MPL have significant hindlimb postural abnormalities when the patella is luxated, despite the lack of bone deformities, but the posture is not different from control when the patella is reduced. These findings suggest that patellar position affects standing posture in the hindlimbs, and that maintaining the patella in a reduced position will maintain normal hindlimb posture in dogs with grade 2 MPL. In contrast to Toy poodles with grade $2 \mathrm{MPL}$, Toy poodles with grade $4 \mathrm{MPL}$ have multiple bone deformities that are likely caused by abnormal muscle traction of the extensor mechanism and abnormal joint alignment due to the persistent medial displacement of the patella. ${ }^{5}$ The effects of MPL on bone morphology may be heightened if the patellar luxation is more severe at a younger age, during the phase of normal bone growth and modelling.

There were no significant differences in the standing flexion/extension angles of the hip, stifle or tarsal joints between the groups, including G2-L. It has been reported that dogs with severe bilateral MPL have a crouched standing posture due to dysfunction of the extensor mechanism of the stifle joint. ${ }^{2,20}$ Unlike dogs with higher grade MPL, this study showed that dogs with grade 2 MPL, whether presently reduced or luxated, do not have changes to the flexion/extension angle of the joints of their hindlimb.

In the G2-L group, the femur was externally rotated compared with the other groups; however, femoral abduction was not different among groups. These results suggest that the hindlimb standing posture is maintained by external rotation of the entire femur, not by abduction of the femur, in the dogs with luxated grade $2 \mathrm{MPL}$. Interestingly, there is no significant difference between the control group and the G2- 
A

B

C

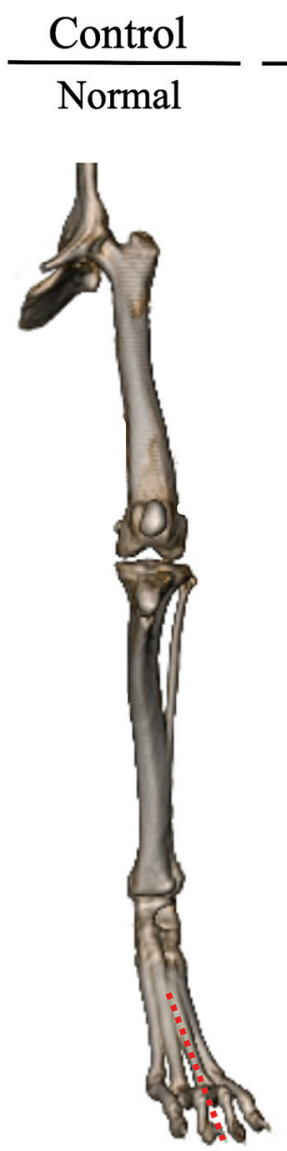

MPL-G2
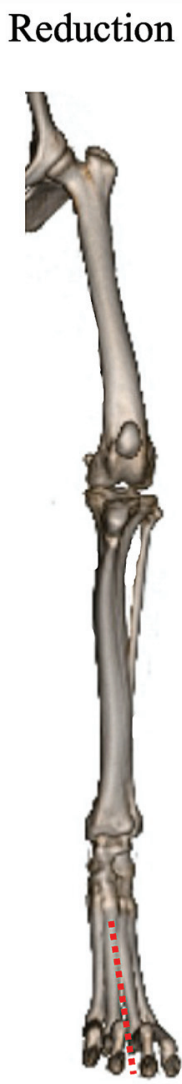

\section{Luxation}

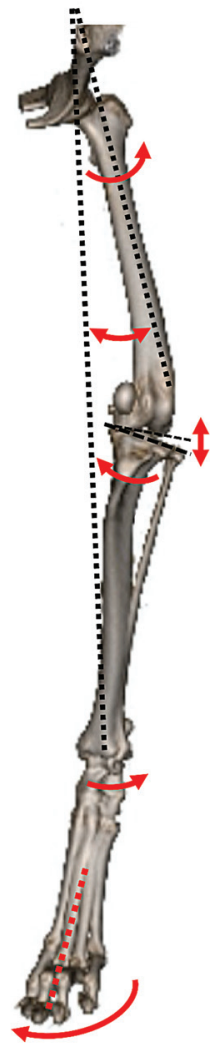

Fig. 10 Awake computed tomography imaging while standing in each group. (A) Control group; normal hindlimb. (B) G2-NL group; hindlimb of MPL-G2 with the patellar reduced. (C) G2-L group: hindlimb of MPL-G2 with luxation of the patella. Significant hindlimb postural abnormalities including external rotation of femur, internal rotation of tibia and foot, external rotation of tarsal joint, large stifle joint convergence angle, genu varum and toe-in standing were observed in the G2-L group (red arrows). MPL-G2, grade 2 medial patellar luxation. G2-NL, reduced grade 2 medial patellar luxation. G2L, luxated grade 2 medial patellar luxation.

NL group, which indicates that reducing of patellar luxation also corrects external femoral rotation.

It has been reported that internal rotation of the proximal tibia relative to the distal femur was observed depending on the severity in dogs with MPL. ${ }^{3}$ As in previous reports, the present study showed that the proximal tibia was internally rotated approximately 30 degrees relative to distal femur in the G2-L group. ${ }^{3}$ In addition, the foot was also significantly internally rotated in the G2-L group compared with the control group, which might simply be a result of the internal rotation of the hindlimb distal to the stifle joint. Interestingly, in the G2-L group, there was a tendency for mild external rotation of the metatarsal bones relative to the tibia. This may be an attempt to correct the malalignment of the entire hindlimb by externally rotating at the tarsal joint to compensate for the internal rotation of

the tibia caused by the medial displacement of the patella. When assessing the limb overall, the dogs in the control group stood in a toe-out posture with approximately 36 degrees of external rotation of the limb, whereas dogs in the G2-L group demonstrated a relative toe-in posture compared with the control group.

Dogs with grade 2 MPL display a genu varum posture when the patella is luxated but not when the patella is reduced, as supported by the larger stifle joint line convergence angle and the larger angle between femoral proximal anatomical axis and the mechanical axis of the hindlimb in G2-L group compared with G2-NL group. In humans, it has been reported that both of these angles increased due to bone deformities in the patients with genu varum and knee osteoarthritis. $^{22,23}$ Conversely, our study has shown that dogs with grade 2 MPL do not have bone deformities but can display genu varum posture when the patella is luxated despite normal bone anatomy. It is likely that the appearance of genu varum in these cases is not caused by femoral abduction, as there was no difference in abduction measurements, but rather by increased femoral external rotation of grade 2 MPL dogs with the patella luxated. Femoral external rotation results in rotation of the distal femur, stifle, and distal limb into an outwardly appearing 'varus' orientation due to normal femoral procurvatum and normal stifle flexion. This is visually supported in -Fig. 10C, where external rotation is evident.

The limitations of this study include a uniform population where only small number of Toy poodles were used and only grade 2 MPL cases were evaluated. The standing posture evaluated in this study might not be 'normal'; therefore, we used the term 'naturally standing' throughout this report. Future studies will be necessary to understand the differences in standing posture in various breeds and various grades of MPL.

In conclusion, awake standing CT imaging was useful in objectively evaluating the hindlimb posture of dogs. Toy poodles with grade 2 MPL had significant hindlimb postural abnormalities when the patella was luxated, which occurred without significant bone deformities. In contrast, no significant postural abnormality was identified in the Toy poodles with grade 2 MPL when the patella was in reduced position. This is the first study to objectively evaluate standing posture in dogs with MPL, and this study contributes to understanding of the pathophysiology of MPL.

Funding

None.

\section{Authors' Contributions}

Y.T. contributed to conception of study, study design, acquisition of data, data analysis and interpretation, drafting or revising of manuscript, approval of submitted manuscript and publicly accountable for relevant content. K.E. contributed to conception of study, study design, data analysis and interpretation, drafting or revising of manuscript, approval of submitted manuscript and publicly accountable for relevant content. A.Y. and K.T. contributed 
to conception of study, acquisition of data, data analysis and interpretation, drafting or revising of manuscript, approval of submitted manuscript and publicly accountable for relevant content. S.T. and K.H. contributed to conception of study, study design, data analysis and interpretation, drafting or revising of manuscript, approval of submitted manuscript and publicly accountable for relevant content. M.S. and K.A. contributed to conception of study, study design, drafting or revising of manuscript, approval of submitted manuscript and publicly accountable for relevant content.

\section{Conflict of Interest}

None declared.

\section{References}

1 LaFond E, Breur GJ, Austin CC. Breed susceptibility for developmental orthopedic diseases in dogs. J Am Anim Hosp Assoc 2002; 38(05):467-477

2 Di Dona F, Della Valle G, Fatone G. Patellar luxation in dogs. Vet Med (Auckl) 2018;9:23-32

3 Žilinčík M, Hluchý M, Takáč L, Ledecký V Comparison of radiographic measurements of the femur in Yorkshire Terriers with and without medial patellar luxation. Vet Comp Orthop Traumatol 2018;31(01):17-22

4 Phetkaew T, Kalpravidh M, Penchome R, Wangdee C. A comparison of angular values of the pelvic limb with normal and medial patellar luxation stifles in Chihuahua dogs using radiography and computed tomography. Vet Comp Orthop Traumatol 2018;31 (02):114-123

5 Yasukawa S, Edamura K, Tanegashima K, et al. Evaluation of bone deformities of the femur, tibia, and patella in Toy Poodles with medial patellar luxation using computed tomography. Vet Comp Orthop Traumatol 2016;29(01):29-38

6 Towle HA, Griffon DJ, Thomas MW, Siegel AM, Dunning D, Johnson A. Pre- and postoperative radiographic and computed tomographic evaluation of dogs with medial patellar luxation. Vet Surg 2005;34(03):265-272

7 Sabharwal S, Zhao C. Assessment of lower limb alignment: supine fluoroscopy compared with a standing full-length radiograph. J Bone Joint Surg Am 2008;90(01):43-51

8 Colebatch AN, Hart DJ, Zhai G, Williams FM, Spector TD, Arden NK. Effective measurement of knee alignment using AP knee radiographs. Knee 2009;16(01):42-45
9 Goodrich ZJ, Norby B, Eichelberger BM, et al. Thoracic limb alignment in healthy labrador retrievers: evaluation of standing versus recumbent frontal plane radiography. Vet Surg 2014;43(07):791-803

10 Usui S, Yasuda H, Koketsu Y. Characteristics of obese or overweight dogs visiting private Japanese veterinary clinics. Asian Pac J Trop Biomed 2016;6:338-343

11 Aulakh KS, Dongaonkar KR, Barnes K, et al. Influence of orthopedic examination on lameness scores and interobserver and intraobserver agreement in dogs with naturally occurring elbow osteoarthritis. Vet Surg 2020;49(03):455-462

12 Fettig AA, Rand WM, Sato AF, Solano M, McCarthy RJ, Boudrieau RJ. Observer variability of tibial plateau slope measurement in 40 dogs with cranial cruciate ligament-deficient stifle joints. Vet Surg 2003;32(05):471-478

13 Jaegger GH, Marcellin-Little DJ, Levine D. Reliability of goniometry in Labrador Retrievers. Am J Vet Res 2002;63(07):979-986

14 Petazzoni M, Jaeger GH. Clinical goniometry, radiographic measurements of the femur. In: Atlas of Clinical Goniometry and Radiographic Measurements of the Canine Pelvic Limb. $2^{\text {nd }}$ Ed. Milano: Merial; 2008:22-32,34-55

15 Hirschmann A, Buck FM, Fucentese SF, Pfirrmann CWA. Upright CT of the knee: the effect of weight-bearing on joint alignment. Eur Radiol 2015;25(11):3398-3404

16 Paley D. Normal lower limb alignment and joint orientation. In: Principles of deformity correction. Berlin, Germany: SpringerVerlag; 2003:1-18

17 Micicoi G, Jacquet C, Sharma A, et al. Neutral alignment resulting from tibial vara and opposite femoral valgus is the main morphologic pattern in healthy middle-aged patients: an exploration of a 3D-CT database. Knee Surg Sports Traumatol Arthrosc 2021;29 (03):849-858

18 Zahn RK, Renner L, Perka C, Hommel H. Weight-bearing radiography depends on limb loading. Knee Surg Sports Traumatol Arthrosc 2019;27(05):1470-1476

19 Patel S, Malhotra K, Cullen NP, Singh D, Goldberg AJ, Welck MJ. Defining reference values for the normal tibiofibular syndesmosis in adults using weight-bearing CT. Bone Joint J 2019;101-B(03):348-352

20 Harasen G. Patellar luxation. Can Vet J 2006;47(08):817-818

21 Newman M, Voss K. Computed tomographic evaluation of femoral and tibial conformation in English Staffordshire Bull Terriers with and without congenital medial patellar luxation. Vet Comp Orthop Traumatol 2017;30(03):191-199

22 Thienpont E, Schwab PE, Cornu O, Bellemans J, Victor J. Bone morphotypes of the varus and valgus knee. Arch Orthop Trauma Surg 2017;137(03):393-400

23 Mondanellli N, Giron F, Losco M, Buzzi R, Aglietti P. Opening wedge high tibial osteotomy using a monoaxial dynamic external fixater. Knee Sports Traumatol Artyrosc 2017;25:306-313 